\title{
Ellagic Acid Protects Dopamine Neurons via Inhibition of NLRP3 Inflammasome Activation in Microglia
}

\author{
Xue-mei He, ${ }^{1,2}$ Yan-zhen Zhou, ${ }^{3}$ Shuo Sheng, ${ }^{1,2}$ Jing-jie Li, ${ }^{1,2}$ Guo-qing Wang, ${ }^{1,2}$ \\ and Feng Zhang $1,2,4$ \\ ${ }^{1}$ Key Laboratory of Basic Pharmacology of Ministry of Education and Joint International Research Laboratory of Ethnomedicine of \\ Ministry of Education, Zunyi Medical University, Zunyi, Guizhou, China \\ ${ }^{2}$ Department of Pharmacology, Key Laboratory of Basic Pharmacology of Guizhou Province and School of Pharmacy, \\ Zunyi Medical University, Zunyi, Guizhou, China \\ ${ }^{3}$ Department of Ear-Nose-Throat Surgery, The Affiliated Hospital of Zunyi Medical University, Zunyi, Guizhou, China \\ ${ }^{4}$ Laboratory Animal Center, Zunyi Medical University, Zunyi, Guizhou, China
}

Correspondence should be addressed to Feng Zhang; zhangfengzmc@163.com

Received 10 July 2020; Revised 20 September 2020; Accepted 5 November 2020; Published 19 November 2020

Academic Editor: Esmael Izadpanah

Copyright (C) 2020 Xue-mei He et al. This is an open access article distributed under the Creative Commons Attribution License, which permits unrestricted use, distribution, and reproduction in any medium, provided the original work is properly cited.

\begin{abstract}
Neuroinflammation plays a crucial role in the pathological process of Parkinson's disease (PD). Nod-like receptor protein 3 (NLRP3) inflammasome was highly located in microglia and involved in the process of neuroinflammation. Activation of the NLRP3 inflammasome has been confirmed to contribute to the progression of PD. Thus, inhibition of NLRP3 inflammasome activation could be an important breakthrough point on PD therapy. Ellagic acid (EA) is a natural polyphenol that has been widely found in soft fruits, nuts, and other plant tissues with anti-inflammatory, antioxidant, and neuroprotective properties. However, the mechanisms underlying EA-mediated anti-inflammation and neuroprotection have not been fully elucidated. In this study, a lipopolysaccharide- (LPS-) induced rat dopamine (DA) neuronal damage model was performed to determine the effects of EA on the protection of DA neurons. In addition, the DA neuronal MN9D cell line and microglial BV-2 cell line were employed to explore whether EA-mediated neuroprotection was through an NLRP3-dependent mechanism. Results indicated that EA ameliorated LPS-induced DA neuronal loss in the rat substantia nigra. Further, inhibition of microglial NLRP3 inflammasome signaling activation was involved in EA-generated neuroprotection, as evidenced by the following observations. First, EA reduced NLRP3 inflammasome signaling activation in microglia and subsequent proinflammatory cytokines' excretion. Second, EA-mediated antineuroinflammation and further DA neuroprotection from LPS-induced neurotoxicity were not shown upon microglial NLRP3 siRNA treatment. In conclusion, this study demonstrated that EA has a profound effect on protecting DA neurons against LPS-induced neurotoxicity via the suppression of microglial NLRP3 inflammasome activation.
\end{abstract}

\section{Introduction}

Parkinson's disease (PD) is a chronic progressive neurodegenerative disease characterized by a deep selective loss of dopamine (DA) neurons in the substantia nigra (SN) [1]. Clinical manifestations include static tremor, slow movement, postural instability, stiffness, and other motor disorders [2]. So far, only a few drugs have been available for PD treatment, and most of them just relieve symptoms and could not prevent the death of DA neurons [3].
It was recognized that age-related excessive oxidative stress led to DA autooxidation, $\alpha$-synuclein accumulation, and glial cell activation [4], which are the main causes of neuroinflammation [5]. Further, neuroinflammation, mediated by microglial activation and infiltrating $\mathrm{T}$ cells at sites of neuronal injury, was considered to be a prominent contributor to the pathogenesis of progressive PD [6]. Microglia are natural immune barriers in the immune system. While stimulated by external conditions, such as brain damage, inflammation, and pathogens, microglia readily become activated and undergo changes in morphology with hypertrophy and 
function with phagocytosis. Most importantly, activated microglia could secrete a large number of proinflammatory factors, such as tumor necrosis factor- $\alpha$ (TNF- $\alpha$ ), interleukin-1 $\beta$ (IL-1 $\beta$ ), and IL-18 [7]. The accumulation of these proinflammatory factors contributes to the progressive loss of DA neurons. However, the neurotoxic factors, such as $\alpha$ synuclein, released by the continuing damaged DA neurons, in turn, induce the secondary activation of microglia, and these activated microglia also secrete proinflammatory factors and further cause DA neuronal loss. Thus, a vicious cycle leading to prolonged neuroinflammation and progressive DA neurodegeneration emerges [8]. Collectively, inhibition of microglia-mediated neuroinflammation holds a promising potential for PD treatment.

Recently, inflammasomes are verified as intracellular proinflammatory pattern recognition receptors (PRRs) to initiate and propagate neuroinflammation. As a large multiprotein complex, inflammasome recruits pro-caspase-1 via ASC (the adaptor molecule apoptosis-associated speck-like protein containing a CARD) and then proceeds to cleave the cytokine precursors, such as pro-IL-1 $\beta$ and pro-IL-18, into mature IL- $1 \beta$ and IL-18 and finally mediates the immune responses against pathogen infection and tissue damage [9]. So far, five different types of inflammasomes have been identified, including NLRP1, NLRP3, NLRC4, Pyrin, and Absent in Melanoma 2 (AIM2) [10]. In particular, the NLRP3 inflammasome has been implicated in the process of neuroinflammation and is highly located in microglia [11]. Increasing evidence revealed that activation of the NLRP3 inflammasome played an important role in the progression of neurodegenerative diseases [12]. Therefore, inhibition of NLRP3 inflammasome activation could be an important breakthrough point in PD therapy.

Ellagic acid (2,3,7,8-tetrahydroxybenzopyrano [5,4,3cde] benzopyran-5-10-dione, EA) is a polyphenol present in many plants, such as pomegranate plants, grapes, raspberries, blackberries, strawberries, and walnuts. EA exhibits a number of pharmacological activities, such as antioxidant and anti-inflammatory effects [13]. Recent studies confirmed that EA exerted neuroprotection against various neurological disorders. For example, EA generated neuroprotection and improved cognitive dysfunctions in a sporadic Alzheimer's disease (AD) animal model [14]. In addition, EA was indicated to confer neuroprotection against ischemic stroke [15]. However, the underlying mechanisms remain unclear. In this study, rat substantia nigral stereotaxic single injection of lipopolysaccharide- (LPS-) elicited DA neuronal loss was performed to investigate EA-exerted neuroprotection and the underlying mechanisms as well. These findings would provide evidence for the future application of EA on $\mathrm{PD}$ treatment.

\section{Materials and Methods}

2.1. Reagents. EA (purity $>95 \%$ ), LPS (Escherichia coli O111:B4), 6-hydroxydopamine (6-OHDA), and apomorphine hydrochloride were obtained from Sigma-Aldrich (St. Louis, CA, USA). Enzyme-linked immunosorbent assay (ELISA) kits for TNF- $\alpha$, IL- $1 \beta$, and IL-18 were bought from
Elabscience Biotechnology Co., Ltd. (Wuhan, China). The MTT assay kit was from Beijing Solarbio Science and Technology Co., Ltd. (Beijing, China). Small interfering RNA (siRNA) against NLRP3 was purchased from GenePharma (Shanghai, China). Anti-CR3 complement receptor (OX-42 Catalog No. Ab1211) and tyrosine hydroxylase (TH, Catalog No. Ab113) antibodies were bought from Abcam (Cambridge, MA, USA). Anti-caspase-1 (Catalog No. 22915-1AP), ionized calcium-binding adapter molecule-1 (Iba-1, Catalog No. 10904-1-AP), $\beta$-actin (Catalog No. 20536-1AP), TNF- $\alpha$ (Catalog No. 17590-1-AP), IL-1 $\beta$ (Catalog No. 66737-1-Ig), IL-18 (Catalog No. 10663-1-AP), rabbit IgG (Catalog No. SA00001-2), and mouse IgG (Catalog No. SA00001-1) antibodies were purchased from the Proteintech Group (Chicago, IL, USA). Anti-NLRP3 (Catalog No. orb101128) antibody was purchased from Biorbyt (Cambridge, United Kingdom).

2.2. Animal and Treatment. Male Wistar rats (200-250 g, 810 weeks) were bought from the Experimental Animal Center in the Third Military Medical University. All experimental procedures were carried out in accordance with the Chinese Guidelines of Animal Care and Welfare, and this study received approval from the Animal Care and Use Committee of Zunyi Medical University (Zunyi, China). Rats were acclimated to their environment for 1 week before the experiments. All the animals were randomly allocated to five experimental groups with six rats in each group: control, EA alone $(50 \mathrm{mg} / \mathrm{kg})$, LPS, LPS+EA $(10 \mathrm{mg} / \mathrm{kg})$, and LPS $+\mathrm{EA}(50 \mathrm{mg} / \mathrm{kg})$. Anesthetized by $7 \%$ chloral hydrate $(0.5 \mathrm{ml} / 100 \mathrm{~g}, v / \mathrm{w})$, rats received a single LPS $(10 \mu \mathrm{g}$ in $5 \mu \mathrm{l}$ $\mathrm{PBS}$ ) unilateral injection into the SN pars compacta followed by the coordinates $5.2 \mathrm{~mm}$ posterior to the bregma, $1.9 \mathrm{~mm}$ lateral to the midline, and $8.0 \mathrm{~mm}$ ventral to the surface of the skull [16]. EA was intragastrically administrated once a day for 7 consecutive days beginning $30 \mathrm{~min}$ before LPS injection. Seven days later, rat behavior changes were analyzed by the rotarod test. Afterwards, animals were sacrificed and the biochemical analysis was performed.

2.3. Rotarod Test. Apomorphine-induced rotation was widely employed for assessing the effects of lesions on the dopaminergic system and the success of treatment in PD animal models. Rats were tested for rotational behavior after receiving an intraperitoneal injection of apomorphine hydrochloride $(0.5 \mathrm{mg} / \mathrm{kg})$. Subsequently, a rotational behavior test was performed by the cylindrical arrangement containing thin steel rods with two parts by compartmentalization to permit the detection of two rats at the same time. Rats were trained to adapt at a speed of $10 \mathrm{rpm} / \mathrm{min}$ before the experiment started, with speed accelerating from 10 to $30 \mathrm{rpm}$ over a period of $5 \mathrm{~min}$ until the rat slid off the steps. The duration time each rat stayed on the rod was recorded and calculated for analyzing the behavior changes of rats [17].

2.4. Immunohistochemical Analysis and Cell Counting in SN. Rat brains were cut with a horizontal sliding microtome into $35 \mu \mathrm{m}$ transverse free-floating sections and immunostained with the corresponding antibodies. Then, brain slices were 
incubated with $0.3 \%$ Triton X-100 and blocked with goat serum. Subsequently, brain slices were incubated with antiTH (1:500), OX-42 (1:800), and NLRP3 $(1: 800)$ antibodies at $4^{\circ} \mathrm{C}$ overnight, respectively [18]. Digital images of $\mathrm{TH}-$ positive neurons, OX-42-positive microglia, and NLRP3positive inflammasomes in midbrain $\mathrm{SN}$ were acquired by an Olympus microscope (Olympus, Tokyo, Japan). Representative fluorescence images were obtained, and the fluorescence intensity was calculated using ipwin32 software.

2.5. Cell Culture and Treatment. The mouse microglial BV-2 cell line was obtained from the China Center for Type Culture Collection (Wuhan, China). Cultures were maintained in minimum essential medium (MEM) supplemented with $10 \%$ heat-inactivated fetal bovine serum (FBS), $100 \mathrm{U} / \mathrm{ml}$ penicillin, and $100 \mu \mathrm{g} / \mathrm{ml}$ streptomycin at $37^{\circ} \mathrm{C}$ in the humidified atmosphere of $5 \% \mathrm{CO}_{2}$ and $95 \%$ air [19]. The DA neuronal MN9D cell line was purchased from the Cell Culture Center in the Institute of Basic Medical Sciences of the Chinese Academy of Medical Sciences (Beijing, China). MN9D cells were cultured in DMEM with 10\% FBS and 1\% penicillin-streptomycin on an atmosphere with $5 \% \mathrm{CO}_{2}$ at $37^{\circ} \mathrm{C}$ in the humidified atmosphere of $5 \% \mathrm{CO}_{2}$ and $95 \%$ air [20].

2.6. MTT Assay. Cell viability was evaluated by MTT assay. BV-2 and MN9D cells were cultured in $1 \times 10^{5} /$ well in 96well plates for $24 \mathrm{~h}$. Afterwards, cells were treated with different concentrations of EA for $30 \mathrm{~min}$ followed by LPS $(100 \mathrm{ng} / \mathrm{ml})$ or 6 -OHDA $(100 \mu \mathrm{M})$ treatment for $24 \mathrm{~h}$ and then incubated with MTT solution ( $5 \mathrm{~g} / \mathrm{l})$ for $4 \mathrm{~h}$. Formazan crystals in the cells were solubilized using $200 \mu$ l dimethyl sulfoxide (DMSO), and the absorbance was detected by an automated microplate reader within a $490 \mathrm{~nm}$ wavelength [21].

2.7. Western Blot Analysis. Total protein content was extracted from rat midbrain tissue and BV-2 cells using a lysis buffer containing protease inhibitors. Protein levels were quantified by BCA [22]. Protein $(10 \mu \mathrm{g})$ from each sample was subjected to SDS-PAGE gel under the reduced conditions. Proteins were then transferred onto polyvinylidene fluoride (PVDF) membranes. The membranes were blocked with $5 \%$ nonfat milk for $2 \mathrm{~h}$ at room temperature and then incubated overnight at $4^{\circ} \mathrm{C}$ with the following primary antibodies: Iba-1 (1:800), NLRP3 (1:800), caspase-1 $(1: 800)$, TNF- $\alpha(1: 1000)$, IL- $1 \beta(1: 500)$, IL-18 $(1: 800)$, and $\beta$-actin $(1: 2000)$. Next, the membranes were incubated for $1 \mathrm{~h}$ with a horseradish peroxidase-conjugated anti-mouse IgG antibody or anti-rabbit IgG at 1:2000 dilution. The blot films were developed with an enhanced ECL Reagent.

2.8. ELISA. The levels of TNF- $\alpha$, IL- $1 \beta$, and IL-18 were measured by ELISA according to the manufacturer's instructions. The microplate reader was used to measure the absorbance at $450 \mathrm{~nm}$.

2.9. Immunofluorescence Staining. Activated microglia were identified with an anti-OX-42 antibody. Cells were fixed with paraformaldehyde (4\%) for $30 \mathrm{~min}$. Later, cells were perme- abilized using Triton X-100 (0.3\%) for $15 \mathrm{~min}$. Then, cells were blocked using a goat serum blocking solution for $60 \mathrm{~min}$ at $37^{\circ} \mathrm{C}$. Thereafter, cells were incubated with $1: 800$ dilution of anti-OX- 42 antibody overnight at $4^{\circ} \mathrm{C}$. Following overnight incubation, cells were incubated in the dark for 30 min with goat anti-rabbit secondary antibody $(1: 1000)$ or goat anti-mouse secondary antibody $(1: 1000)$. Cells were also counterstained with DAPI for 5 min [23]. After rinsing cells with PBS, representative fluorescence images were obtained using an EVOS ${ }^{\circledR}$ Floid $^{\circledR}$ Cell Imaging Station. The fluorescence intensity was calculated by using ipwin 32 software.

2.10. RNA Transfection. BV-2 cells were cultured and seeded in a 6-well plate at a density of $1 \times 10^{5}$ cells $/ \mathrm{ml}$. The transfection of siRNA was performed complying with the manufacturer's protocol. A total of $2 \mu \mathrm{l}$ of NLRP3 siRNA was diluted into $18 \mu \mathrm{l}$ of transfection medium. GP-siRNA-Mate plus $(180 \mu \mathrm{l})$ was used to transfect with siRNA dilution $(20 \mu \mathrm{l})$. After transfection for $6 \mathrm{~h}$, the transfection solution was removed and cells were rinsed with PBS and replaced with MEM containing 2\% FBS and then treated with EA for $30 \mathrm{~min}$ followed by LPS $(100 \mathrm{ng} / \mathrm{ml})$ treatment for $24 \mathrm{~h}$ [19]. The gene sequences were as follows: sense, $5^{\prime}$-UUC UCC GAA CGU GUC ACG UTT- $3^{\prime}$; antisense, $5^{\prime}$-ACG UGA CAC GUU CGG AGA ATT-3'.

2.11. Statistical Analysis. Results were indicated as the mean \pm standard error of the mean (SEM). Statistical significance was analyzed by one-way analysis of variance (ANOVA) using GraphPad Prism software (GraphPad Software Inc., San Diego, CA, USA). Upon ANOVA demonstrating the significant differences, pairwise comparison between means was evaluated by Bonferroni's post hoc test with correction. A value of $p<0.05$ was considered statistically significant.

\section{Results}

3.1. EA Attenuated LPS-Induced DA Neuronal Damage in SN In Vivo. Neuroprotective effects of EA on LPS-induced DA neuronal damage were investigated in rats. As shown in Figure 1(a), LPS reduced the time rats stayed on the rod, compared with the control group. However, EA $(50 \mathrm{mg} / \mathrm{kg})$ attenuated the LPS-caused decrease in the time rats remained on the rod, while no significant protection of EA $(10 \mathrm{mg} / \mathrm{kg})$ was discerned. To further confirm EA-mediated DA neuroprotection, the TH-positive neuronal number and $\mathrm{TH}$ protein expression were determined. As shown in Figure 1(b), EA $(50 \mathrm{mg} / \mathrm{kg})$ ameliorated the LPS-induced decrease in $\mathrm{TH}$ protein expression. Consistent with $\mathrm{TH}$ protein detection results, TH-positive neuronal counting indicated that EA exerted neuroprotection against LPS-induced DA neuronal loss (Figure 1(c)).

3.2. EA Ameliorated LPS-Elicited Activation of Microglia and NLRP3 Inflammasome Signaling In Vivo. Next, the effects of EA on microglia and NLRP3 inflammasome activation were investigated. To verify the connection between the NLRP3 inflammasome and microglia, the double-immunofluorescence 


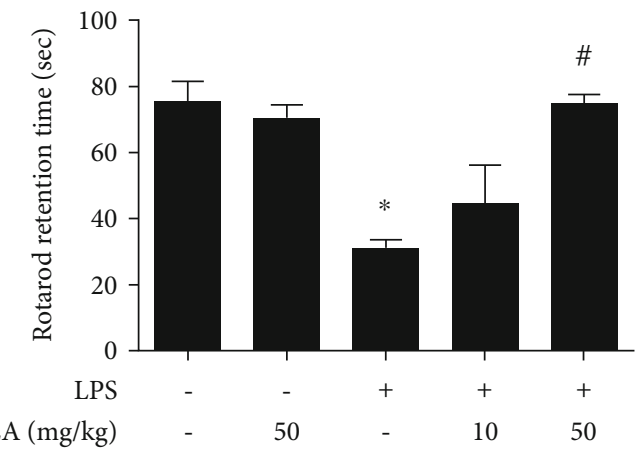

(a)
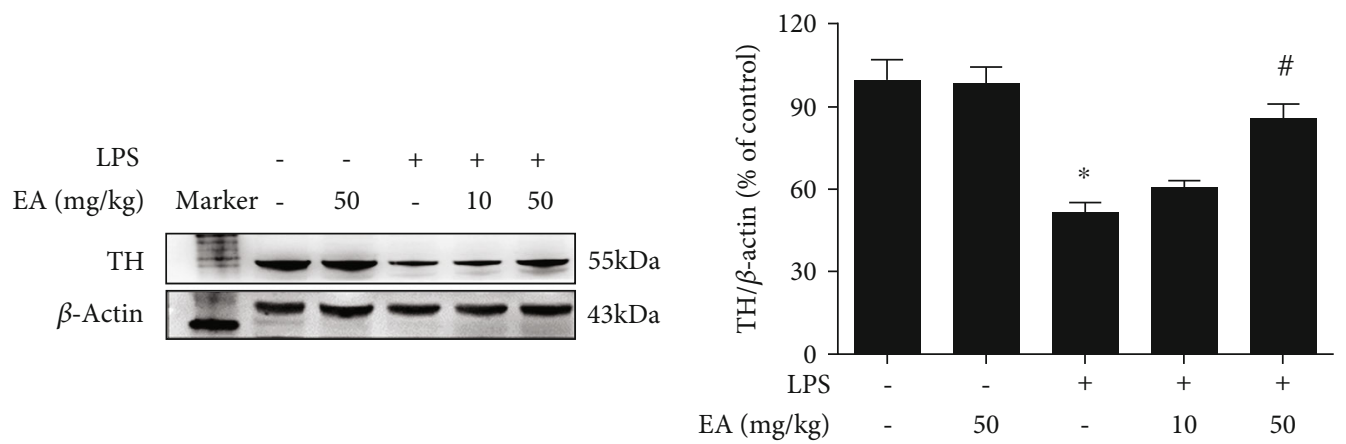

(b)
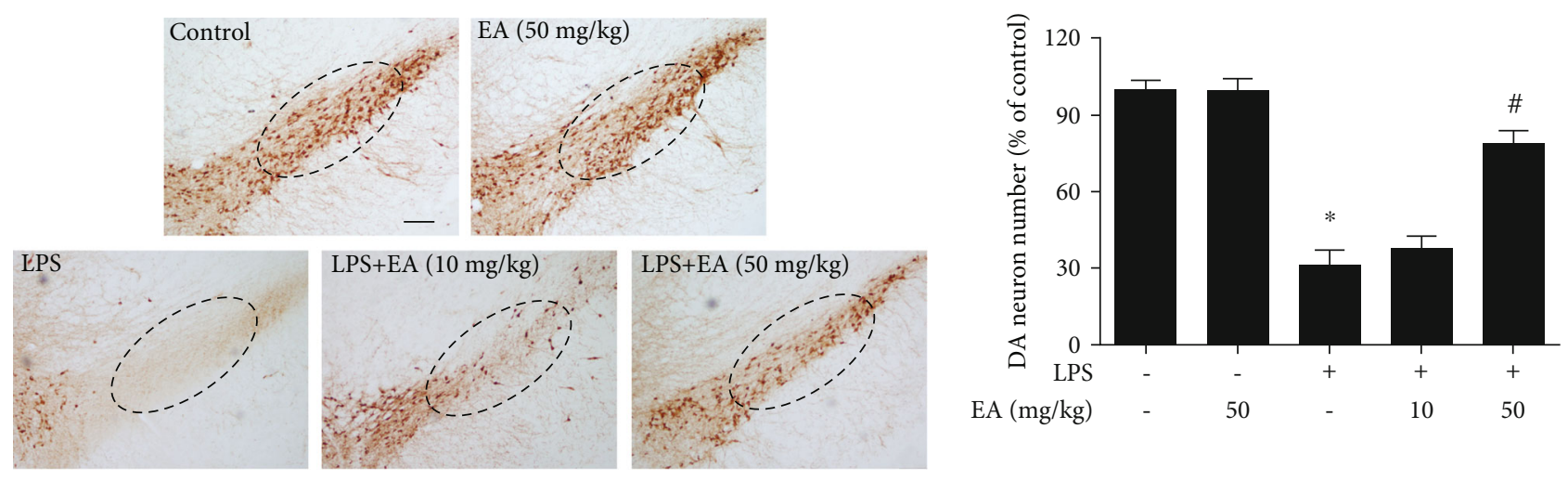

(c)

FIgURE 1: EA attenuated LPS-induced DA neuronal damage in SN in vivo. Rats were intragastrically given EA (50 mg/kg) for 7 consecutive days. Rat behavior changes were analyzed by the rotarod test (a). TH protein expression in the rat midbrain was tested by western blot assay (b). Brain sections were immunostained with an anti-TH antibody, and the number of TH-positive neurons in SN was counted (c). The "ellipse" presented the area of SN. Scale bar $=200 \mu \mathrm{m}$. Data were the mean \pm SEM from 6 rats. * $p<0.05$ compared with the control group; $\# p<0.05$ compared with the LPS group.

calibration site was conducted. As shown in Figure 2(a), the NLRP3 inflammasome was activated and located in activated microglia. EA attenuated LPS-induced activation of the NLRP3 inflammasome in microglia. Also, EA inhibited Iba1 protein expression induced by LPS (Figure 2(b)). In addition, EA suppressed LPS-induced activation of NLRP3 inflammasome signaling (Figure 2(c)) and proinflammatory cytokine (IL- $1 \beta$, TNF- $\alpha$, and IL-18) protein expressions (Figure 2(d)).

3.3. EA Had No Direct Neuroprotective Effects on DA Neurons. To further confirm whether EA produced direct neuroprotective actions on DA neurons, the effects of EA on 6-OHDA-induced DA neuronal damage in vitro were determined. First, as shown in Figure 3(a), 6-OHDAinduced neurotoxicity was not attenuated by EA treatment in MN9D cell-enriched cultures. Next, in TH-positive neuronal counting analysis (Figures 3(b) and 3(c)), 6-OHDA caused TH-positive neuronal loss and EA did not confer protection from 6-OHDA-induced neuronal damage. Similar results were indicated in the $\mathrm{TH}$ protein detection shown in Figure 3(d). These results demonstrated that EA did not generate direct neuroprotection on DA neurons.

3.4. EA Inhibited Microglial NLRP3 Inflammasome Activation In Vitro. The effects of EA on microglia and 

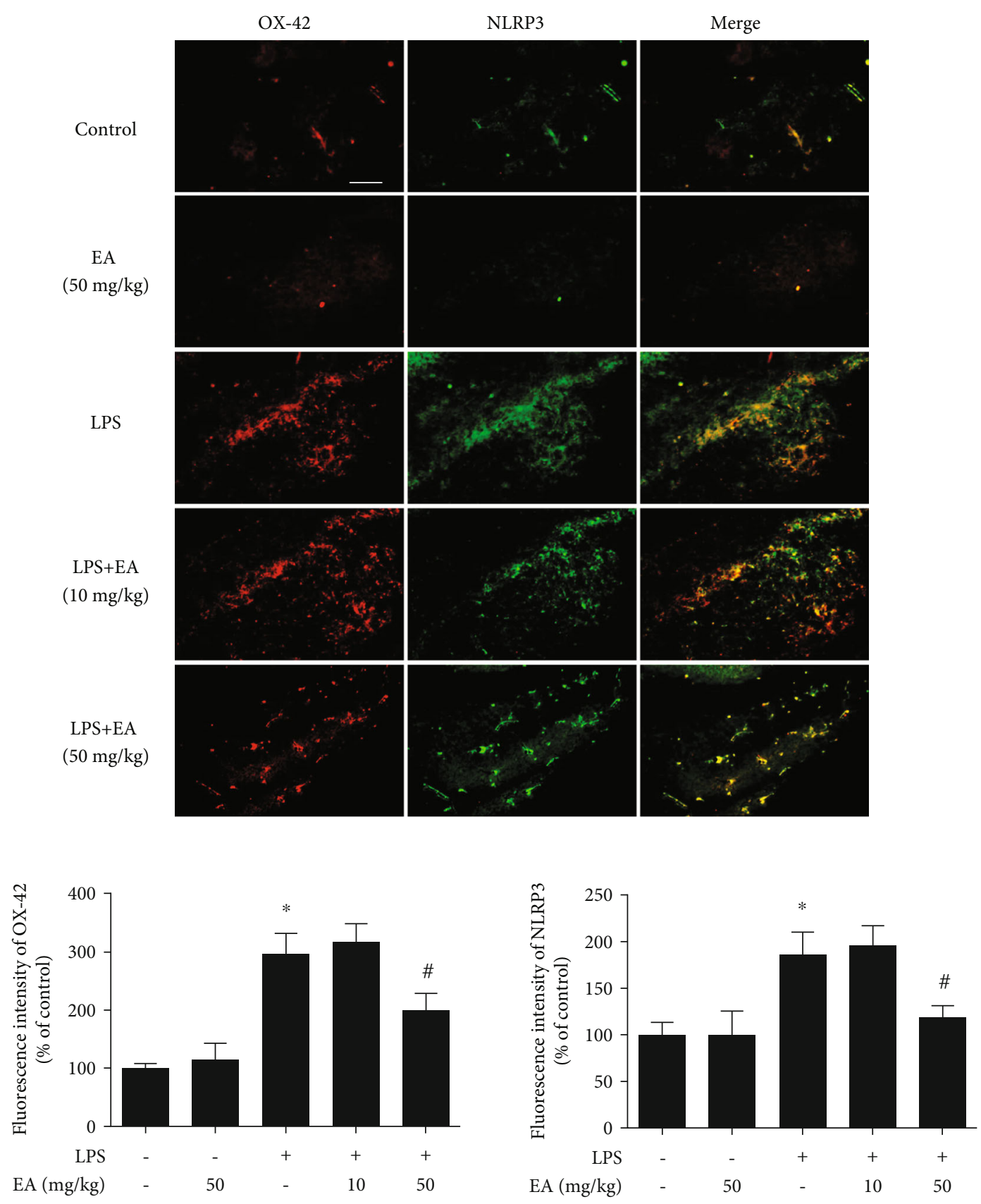

(a)
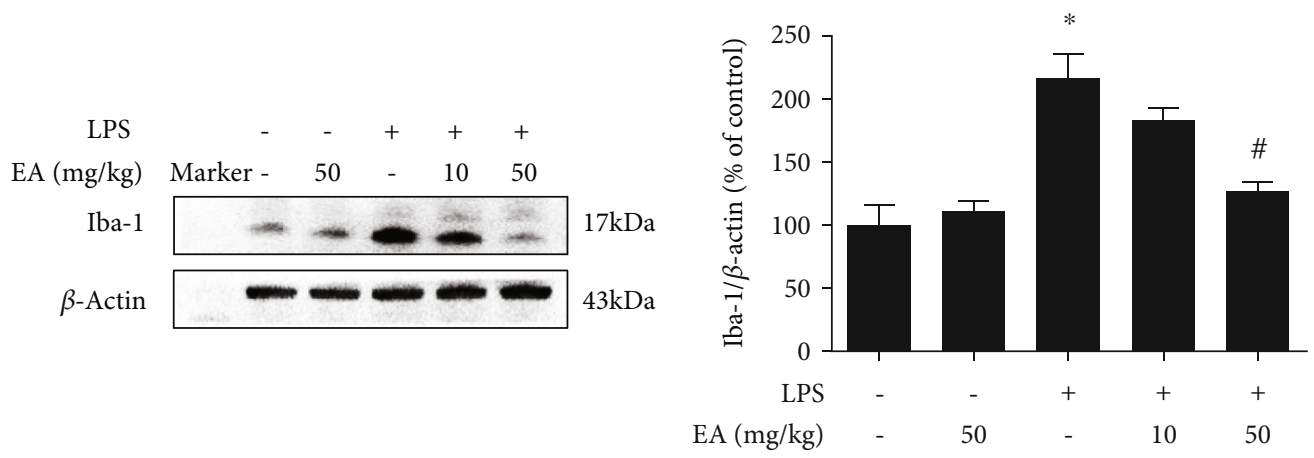

(b)

Figure 2: Continued. 

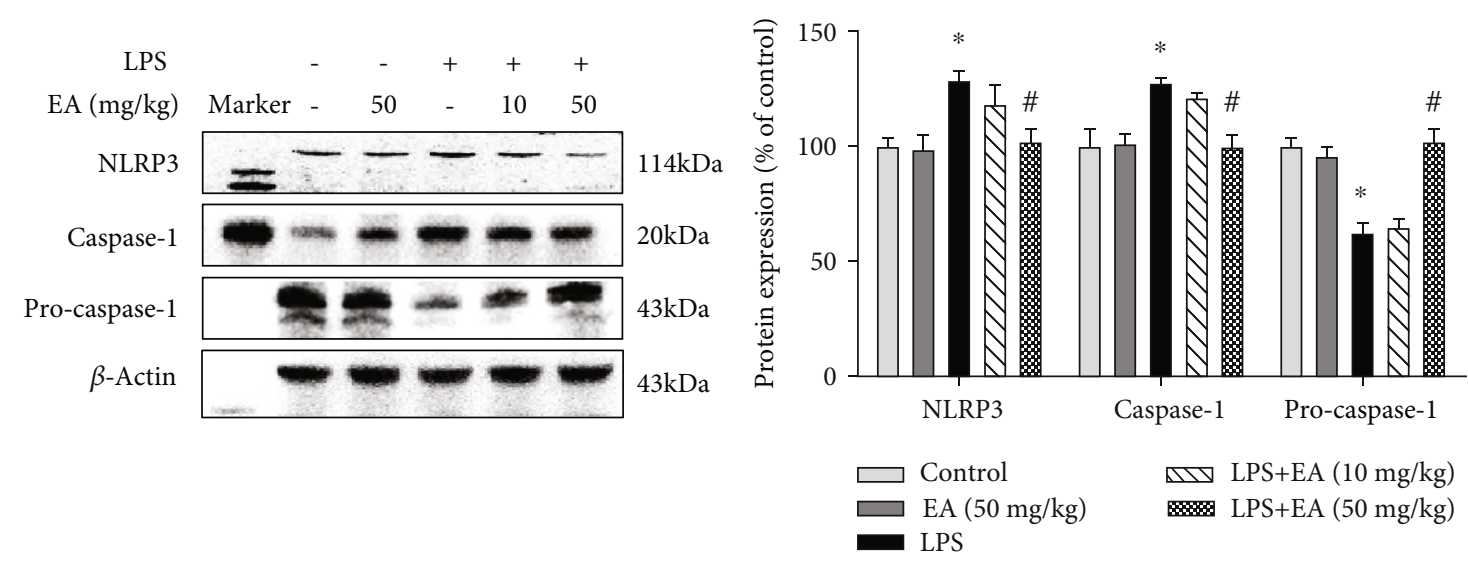

(c)
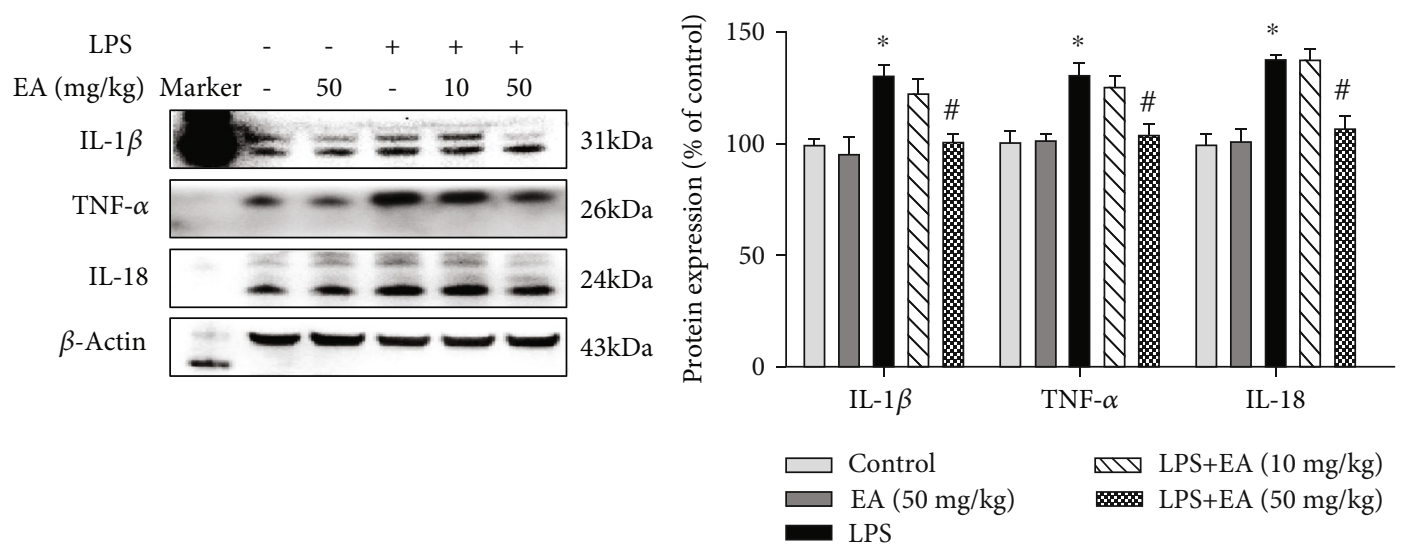

(d)

FIGURE 2: EA ameliorated LPS-elicited activation of microglia and NLRP3 inflammasome signaling in vivo. Rat brains were collected and stained by double immunofluorescence with anti-NLRP3 and anti-OX-42 antibodies (green fluorescence represented NLRP3 inflammasome, and red fluorescence represented microglia) (a). The protein expressions of Iba-1 (b); NLRP3, caspase-1, and pro-caspase1 (c); and TNF- $\alpha$, IL- $1 \beta$, and IL-18 (d) in the rat midbrain were determined via western blot assay. Data were the mean \pm SEM from 6 rats and expressed as a percentage of the control group. ${ }^{*} p<0.05$ compared with the control group; ${ }^{*} p<0.05$ compared with the LPS group.

NLRP3 inflammasome signaling activation were further confirmed in vitro. First, BV-2 cells were employed to examine the effects of EA on LPS-induced microglial activation. As shown in Figure 4(a), immunofluorescence staining assay indicated that EA reduced LPS-induced microglial activation. Also, EA decreased LPS-induced higher protein expression of Iba-1 (Figure 4(b)). Then, EA inhibited the activation of microglial NLRP3 inflammasome signaling induced by LPS (Figure 4(b)). In addition, EA eliminated LPS-induced production of proinflammatory factors, such as TNF- $\alpha$, IL- $1 \beta$, and IL-18, in the culture medium (Figure 4(c)).

3.5. NLRP3 Inflammasome Signaling Inactivation Was Involved in EA-Mediated Anti-Inflammatory Properties. To investigate the role of NLRP3 inflammasome signaling in EA-mediated antineuroinflammation, NLRP3 siRNA was performed in BV-2 cell cultures. First, as shown in Figure 5(a), NLRP3 siRNA was transfected into BV-2 cells and the successful transfection with NLRP3 siRNA was evaluated by the NLRP3 protein level. After NLRP3 siRNA administration, both NLRP3 siRNA and EA inhibited LPSinduced NLRP3 inflammasome signaling activation. How- ever, no significant difference of NLRP3 and caspase-1 protein expressions between the LPS+EA and LPS+EA+NLRP3 siRNA groups was discerned (Figure 5(b)). In addition, the effects of EA on proinflammatory factors' excretion with NLRP3 siRNA application were measured. In parallel with NLRP3 inflammasome signaling analysis, EA did not reduce LPS-induced release of proinflammatory factors again after NLRP3 siRNA treatment (Figure 5(c)). These observations indicated EA attenuated microglia-induced neuroinflammation via inhibition of NLRP3 inflammasome signaling activation.

3.6. EA Targeted Microglial NLRP3 Inflammasome to Produce DA Neuroprotection. Since microglia were the target of EA-generated DA neuroprotection, whether this neuroprotection resulted from inhibiting microglial NLRP3 inflammasome activation was then explored. As shown in Figure 6, compared with the MCM (LPS) group, both MCM (LPS+NLRP3 siRNA) and MCM (LPS+EA) protected against MCM (LPS)-induced neurotoxicity evidenced by cell viability and $\mathrm{TH}$ protein expression detection, whereas no significant difference of neuroprotection between these two 

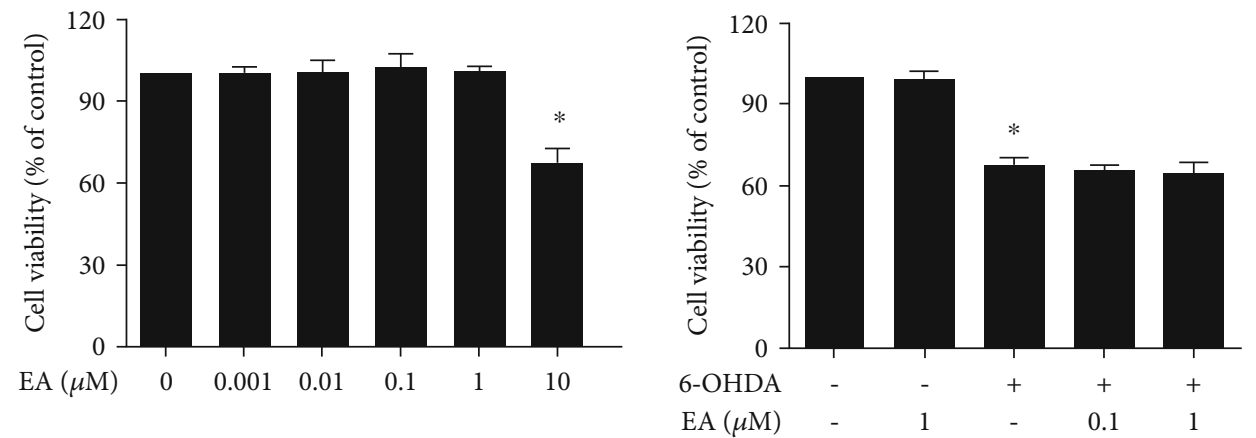

(a)

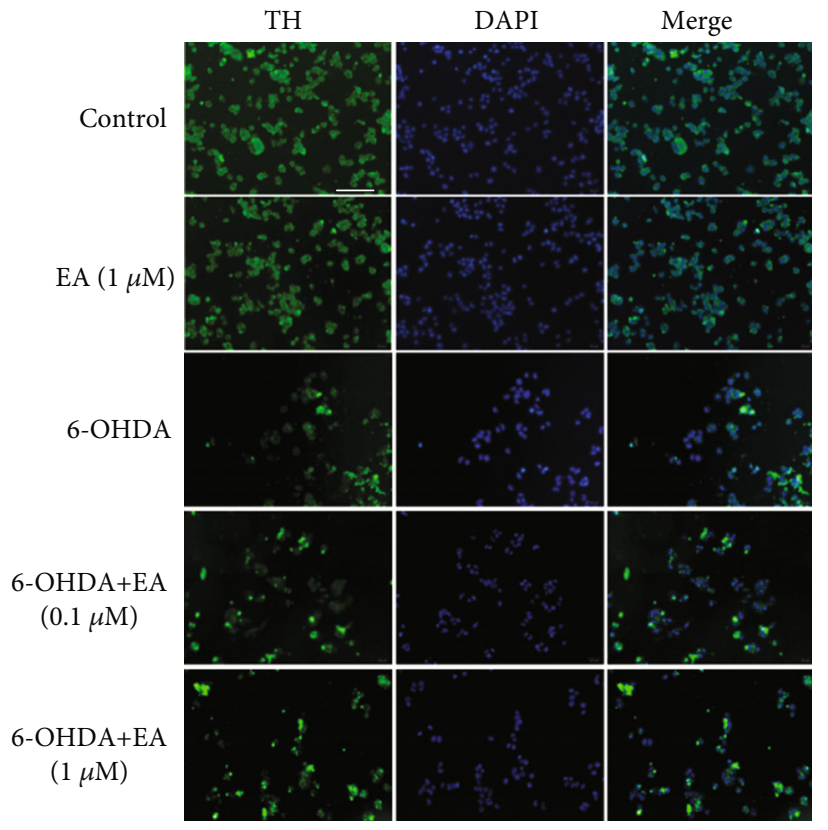

(b)

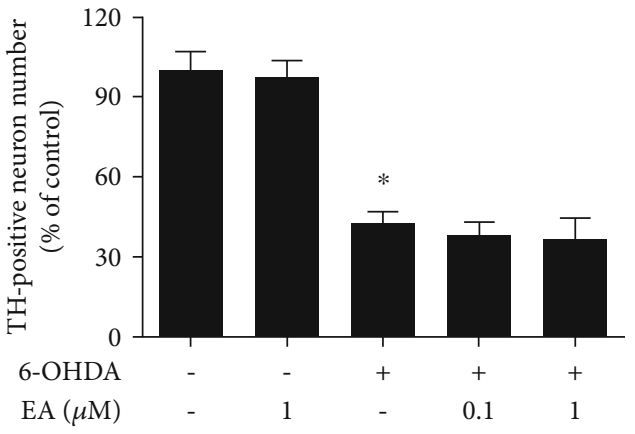

(c)
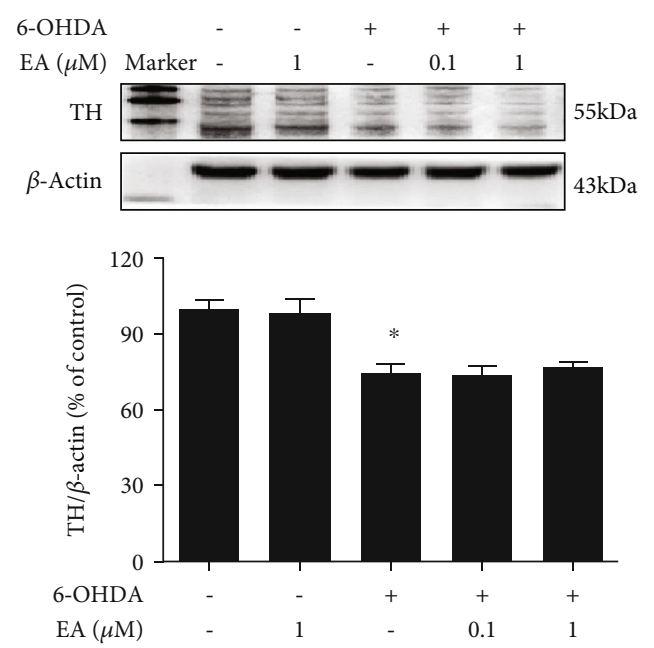

(d)

Figure 3: EA had no direct neuroprotective effects on DA neurons. MN9D cells were treated with EA ( 0.1 and $1 \mu \mathrm{M})$ for 30 min and then incubated with 6-OHDA $(100 \mu \mathrm{M})$ for $24 \mathrm{~h}$. Cell viability was determined by MTT assay (a). 6-OHDA-induced MN9D cell damage was evaluated by immunostaining (b) and cell counting (c). Scale bar $=100 \mu \mathrm{m}$. The protein expression of TH was detected by western blot assay (c). Data were the mean \pm SEM from three independent experiments performed in triplicate. ${ }^{*} p<0.05$ compared with control cultures; ${ }^{*} p<0.05$ compared with 6-OHDA-treated cultures. 


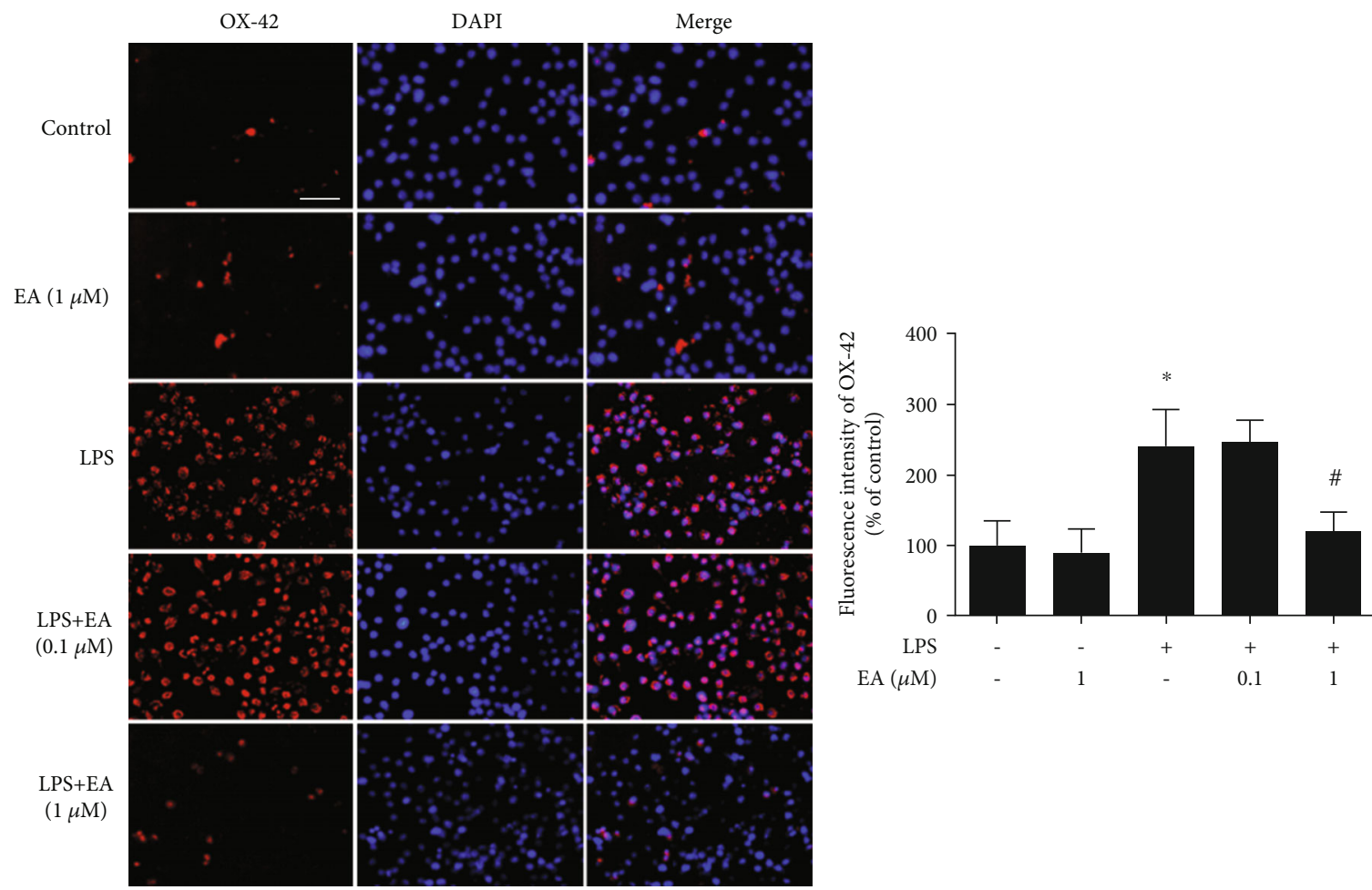

(a)
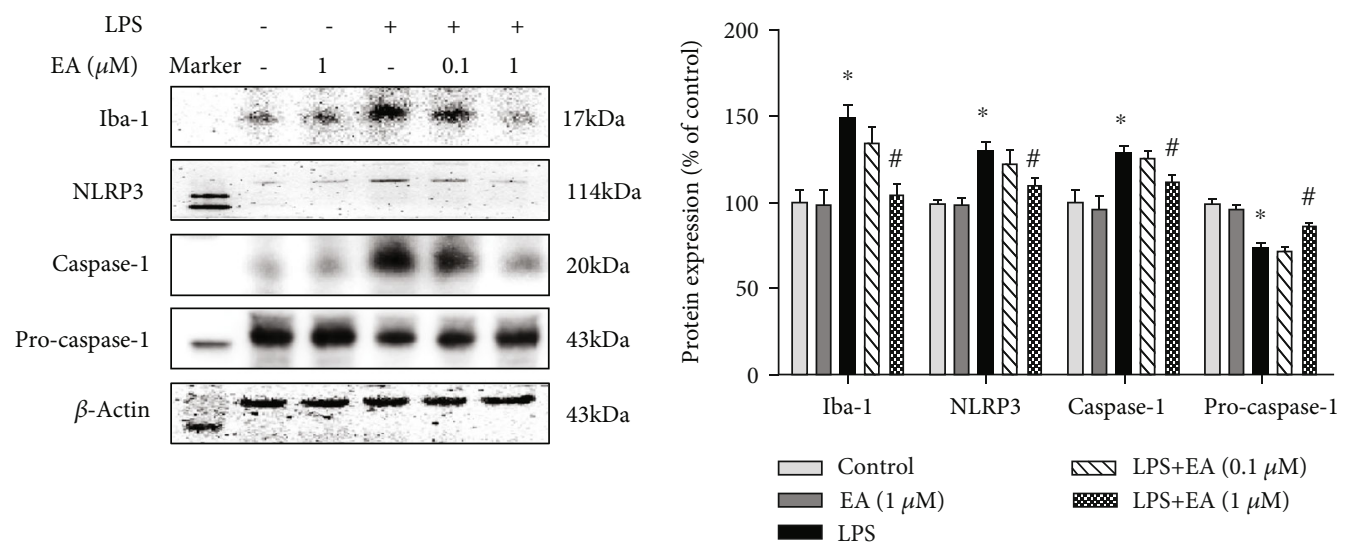

(b)
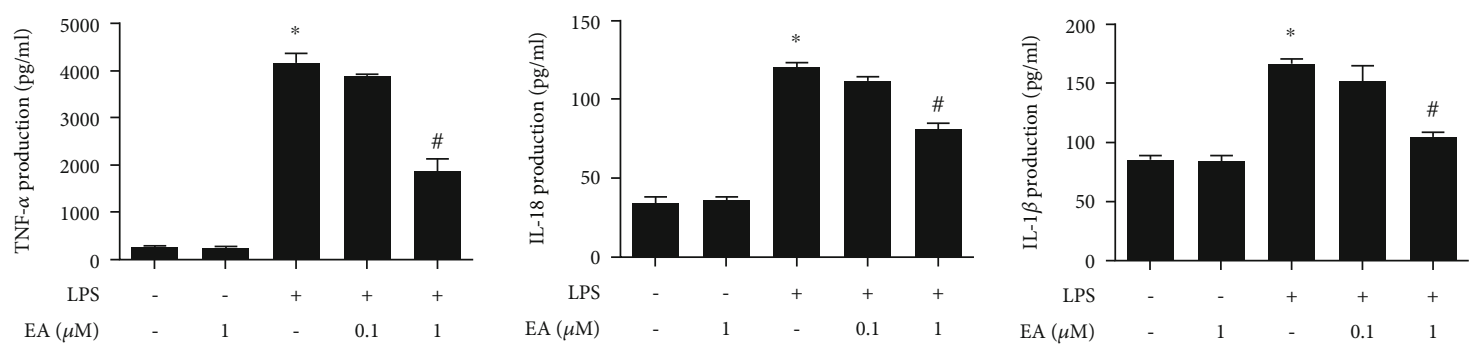

(c)

FIGURE 4: EA inhibited microglial NLRP3 inflammasome activation in vitro. BV-2 cells were treated with EA (0.1 and $1 \mu \mathrm{M})$ for 30 min and then incubated with LPS $(100 \mathrm{ng} / \mathrm{ml})$ for $24 \mathrm{~h}$. Microglial activation was evaluated by immunostaining with an anti-OX-42 antibody (a) and quantitated by western blot analysis with an anti-Iba-1 antibody (b). Scale bar $=100 \mu \mathrm{m}$. The effects of EA on NLRP3 inflammasome signaling activation in BV-2 cells were detected via western blotting (b). The ratios of densitometry values of Iba-1, NLRP3, caspase-1, and pro-caspase1 with $\beta$-actin were analyzed and normalized to each respective control cultures. The release of proinflammatory factors, such as TNF- $\alpha$, IL$1 \beta$, and IL-18, in BV-2 cell culture medium was measured by ELISA (c). Data were the mean \pm SEM from three independent experiments performed in triplicate. ${ }^{*} p<0.05$ compared with control cultures; ${ }^{\#} p<0.05$ compared with LPS-treated cultures. 

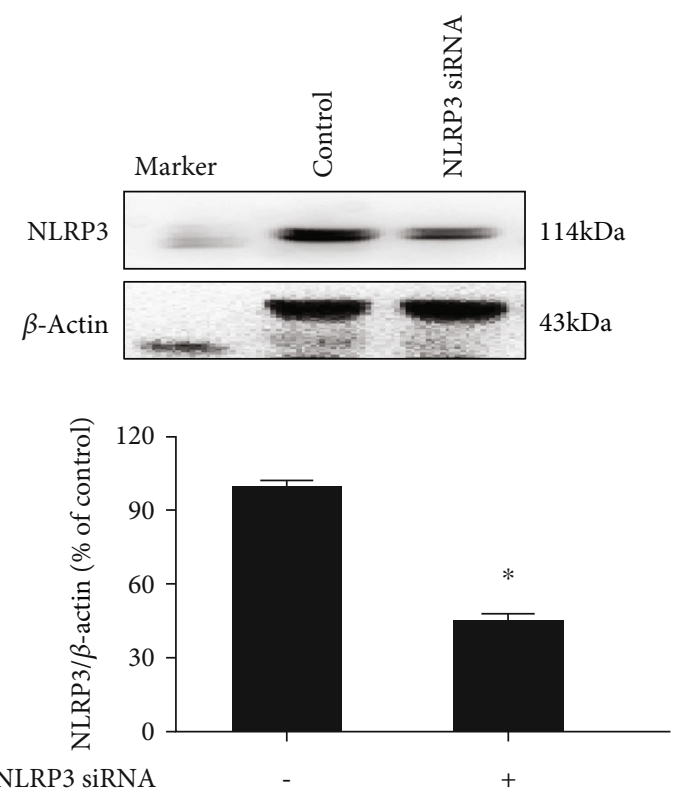

NLRP3 siRNA

(a)
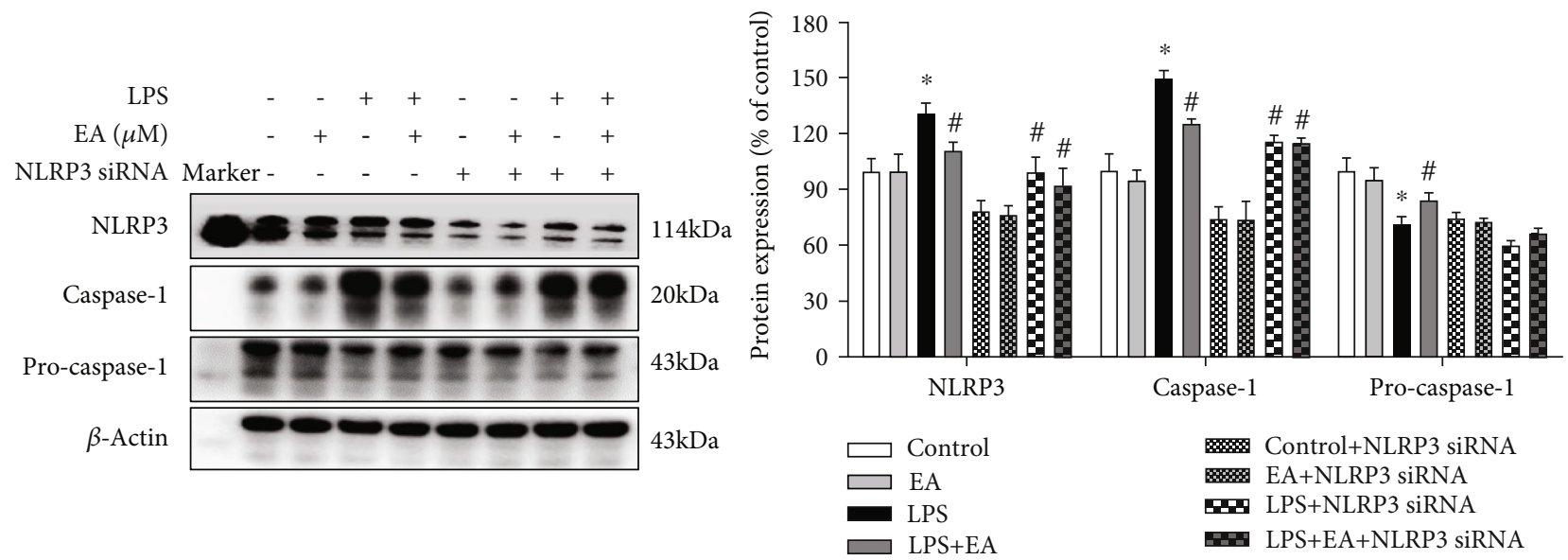

(b)

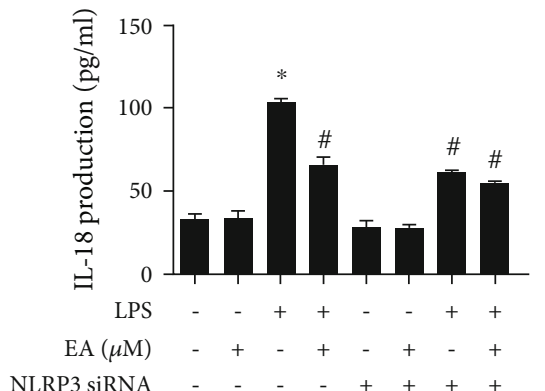

NLRP3 siRNA

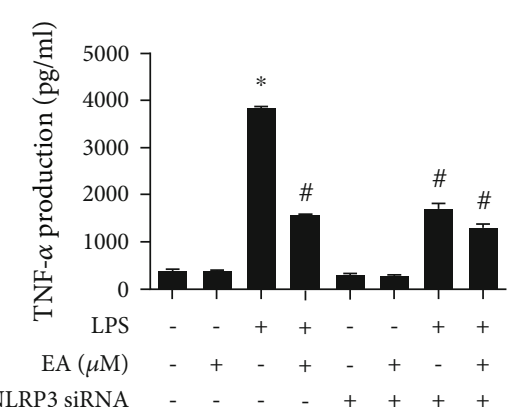

(c)

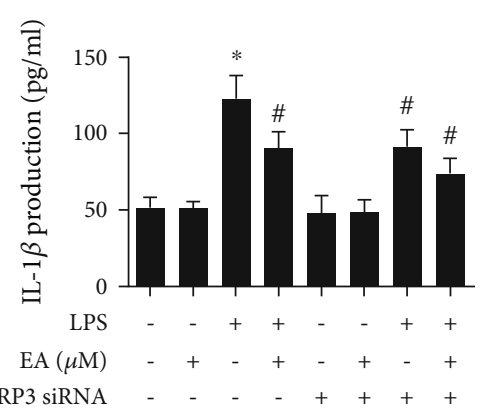

NLRP3 siRNA

FIGURE 5: NLRP3 inflammasome signaling inactivation was involved in EA-mediated anti-inflammatory properties. BV-2 cells were treated with NLRP3 siRNA ( $40 \mathrm{nM})$. After $6 \mathrm{~h}$ of transfection, the transfection solution was removed and cells were rinsed with PBS. The silencing efficiency was assessed via NLRP3 protein expression detection (a). Moreover, BV-2 cells were treated with EA ( $1 \mu \mathrm{M})$ in the presence of NLRP3 siRNA and then exposed to LPS for $24 \mathrm{~h}$. The protein expressions of Iba-1, NLRP3, caspase-1, and pro-caspase-1 in BV-2 cells were detected via western blot assay (b). The levels of TNF- $\alpha$, IL-1 $\beta$, and IL-18 in the culture medium were measured by ELISA (c). Data were the mean \pm SEM from three independent experiments performed in triplicate. ${ }^{*} p<0.05$ compared with control cultures; ${ }^{\#} p<0.05$ compared with LPS-treated cultures. 

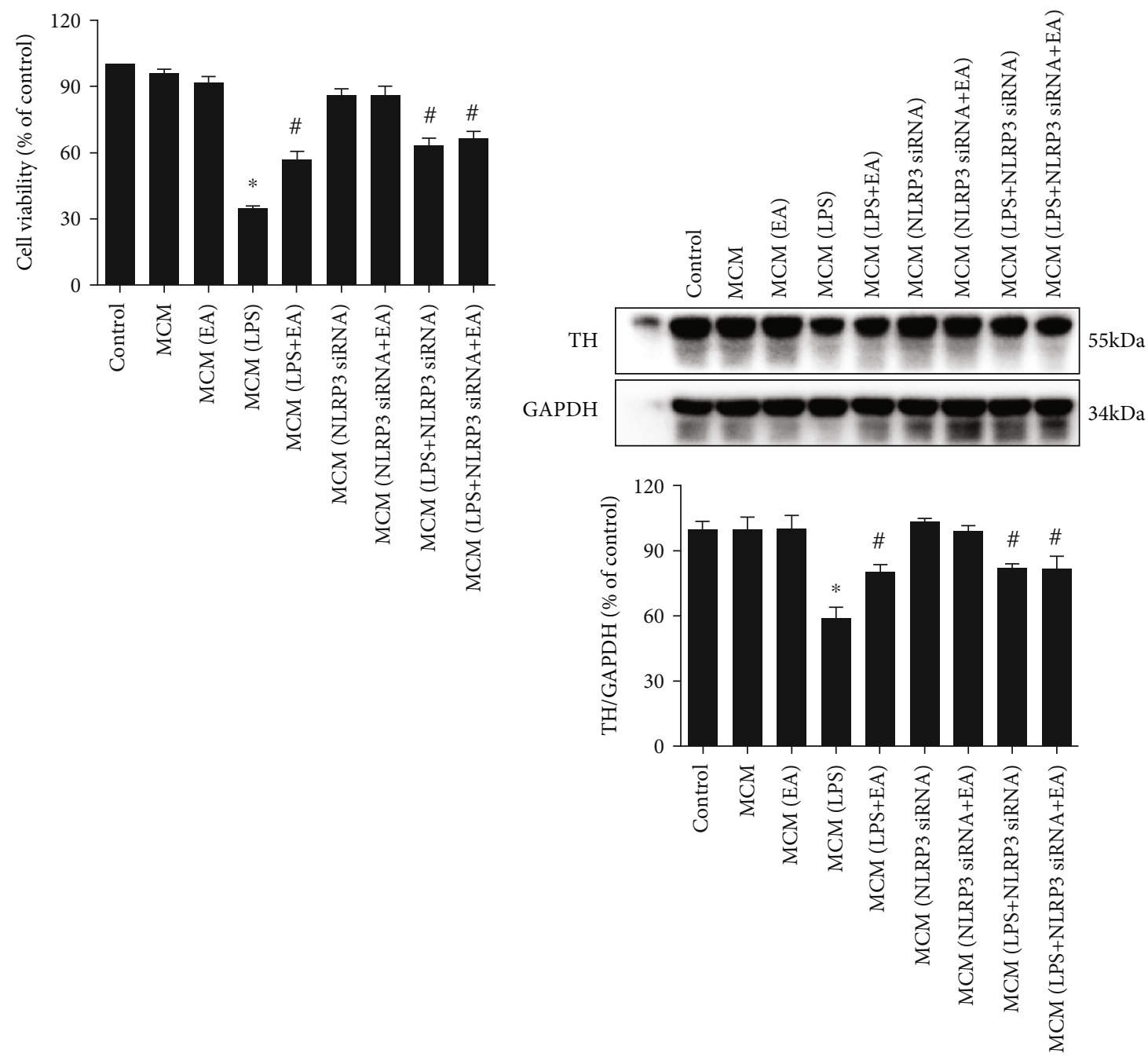

(a)

(b)

FIGURE 6: EA targeted microglial NLRP3 inflammasome to produce DA neuroprotection. Microglia-conditioned medium (MCM) prepared from BV-2 cell cultures with administration of EA (MCM (EA)), LPS (MCM (LPS)), LPS+EA (MCM (LPS+EA)), NLRP3 siRNA (MCM (NLRP3 siRNA)), NLRP3 siRNA+EA (MCM (NLRP3 siRNA+EA)), NLRP3 siRNA+LPS (MCM (NLRP3 siRNA+LPS)), and LPS+NLRP3 siRNA+EA (MCM (LPS+NLRP3 siRNA+EA)) was harvested and added to MN9D cells incubated for $24 \mathrm{~h}$. MN9D cell viability was determined by MTT assay (a). TH protein expression was tested by western blot assay (b). Data were the mean \pm SEM from three independent experiments performed in triplicate. ${ }^{*} p<0.05$ compared with control cultures; ${ }^{*} p<0.05$ compared with MCM (LPS)-treated cultures.

groups was exhibited. Further, MCM (LPS+NLRP3 siRNA $+\mathrm{EA})$ did not exert more DA neuroprotection against MCM (LPS)-caused neuronal injury than MCM (LPS +NLRP3 siRNA) or MCM (LPS+EA) treatment.

\section{Discussion}

The present study is aimed at investigating the neuroprotective actions of EA on LPS-induced DA neuronal loss and evaluating the role of microglia-mediated neuroinflammation in this neuroprotection. Results indicated that EA protected DA neurons against LPS-induced neurotoxicity in SN. Further, inhibition of microglial NLRP3 inflammasome signaling activation was involved in EA-generated neuroprotection, as evidenced by the following observations. First, EA reduced NLRP3 inflammasome signaling activation in microglia and subsequent proinflammatory cytokines' excretion. Second, EA-mediated antineuroinflammation and further DA neuroprotection from LPS-induced neurotoxicity were not shown upon microglial NLRP3 siRNA treatment. Taken together, EA conferred neuroprotection against LPSinduced DA neuronal damage via inhibition of microglial NLRP3 inflammasome signaling activation (Figure 7).

Neuroinflammation is considered to be the most common feature of the aging brain and neurodegenerative disorders, including $\mathrm{AD}, \mathrm{PD}$, and amyotrophic lateral sclerosis (ALS) [5]. It is primarily mediated by activated glial cells, especially for microglia, and accompanied by the secretion of proinflammatory mediators [24]. As the first defense of immune surveillance, microglia readily become activated and predominately participate in the inflammatory response. In addition, astroglia could amplify microglia-mediated 


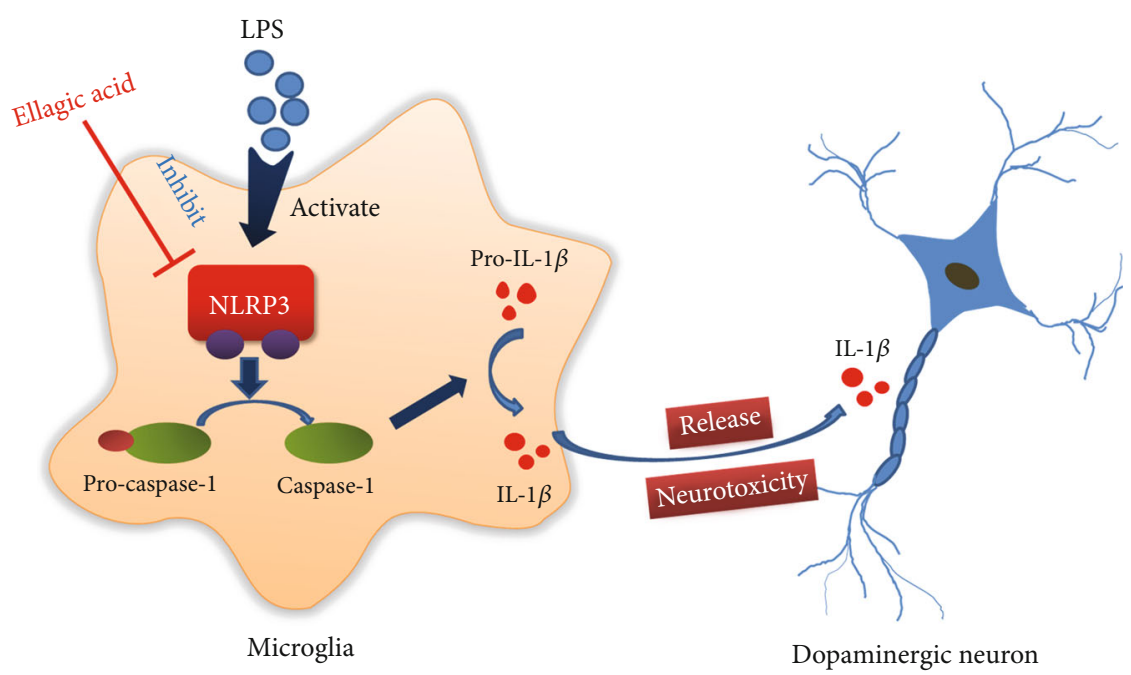

FIgURE 7: The mechanisms underlying EA-mediated dopamine neuroprotection.

neuroinflammation and result in the feedback loop of neuroinflammatory reactions [25]. In this regard, understanding the molecular mechanisms of PD can be found by investigating microglia-mediated neuroinflammation [26]. Therefore, to target microglia-derived proinflammatory cytokines might offer promising therapeutic approaches for PD management. In this study, EA protected DA neurons against LPS-induced neurotoxicity and reduced microglial proinflammatory factors' release, suggesting that EA-generated DA neuroprotection was closely associated with the inhibition of microgliamediated neuroinflammation. Consistent with this finding, EA was previously confirmed to reduce the production of the proinflammatory cytokines, such as TNF- $\alpha$ and IL-6, in LPS-stimulated RAW 264.7 macrophage cells [27]. Furthermore, the dose of EA $(50 \mathrm{mg} / \mathrm{kg})$ presented beneficial effects on protection against LPS-induced DA neurotoxicity and inhibition of microglial NLRP3 inflammasome activation, whereas significant effects mediated by EA $(10 \mathrm{mg} / \mathrm{kg})$ were shown. This dose profile suggested that EA $(10-50 \mathrm{mg} / \mathrm{kg})$ was under the effective dose range in rats and guided that the selection of doses of EA complied with the dose-effect relationship. However, additional clinical studies are indeed needed to validate the dose range and dose-effect relationship of EA on humans corresponding to that in rats.

In addition, inflammasomes are multiprotein complexes responsible for intracellular sensors of environmental and cellular stress $[28,29]$. As the inflammasome family member, the NLRP3 inflammasome is mainly involved in the response of neuroinflammation [11]. Upon cellular stress, assembly of NLRP3 inflammasome triggers caspase-1 activation and further caspase-1-mediated production of IL- $1 \beta$ and IL-18, thereby initiating neuroinflammation [30]. In PD patient brains, the NLRP3 inflammasome was potentially activated by insoluble $\alpha$-synuclein aggregates and oxidative stress [31]. However, deficiency of NLRP3 inflammasome attenuated motor dysfunction and DA neurodegeneration in a PD mouse model [32]. Thus, inhibition of NLRP3 inflammasome activation might be beneficial for PD intervention. The present study demonstrated that EA could inhibit
NLRP3 inflammasome signaling activation. Similar results exhibited that EA prevented monocrotaline-induced rat pulmonary artery hypertension via inhibiting the activation of the NLRP3 inflammasome and caspase-1 in the lungs and release of proinflammatory cytokines, such as IL- $\beta$, in serum [33]. On the other hand, this study indicated that EAmediated DA neuroprotection was present in neuronmicroglia cocultures but not in neuron-enriched cultures, implying that microglia were at least essential for EAgenerated neuroprotection. Moreover, EA was found to reduce LPS-induced proinflammatory factors' production. Collectively, EA-inhibited microglial activation, and the subsequent proinflammatory factors' release might be attributed to the inhibition of microglial NLRP3 inflammasome activation. This conclusion was verified by the following observations: (1) EA inhibited microglial NLRP3 and pro-caspase-1 activation and IL-1 $\beta$ production; (2) EA could not further suppress LPS-induced proinflammatory factors' release and produce DA neuroprotection after neuron-microglia cocultures treated by NLRP3 siRNA.

Interestingly, EA not only decreased IL- $\beta$ and IL-18 production via inhibition of NLRP3 inflammasome activation but also reduced TNF- $\alpha$ secretion. Why did EA suppress TNF- $\alpha$ production during EA-inhibited NLRP3 inflammasome activation? The amount of evidence indicated that the NLRP3 inflammasome participated in NF- $\kappa \mathrm{B}$-mediated inflammatory processes of diseases $[34,35]$, which suggested that there was crosstalk between the NLRP3 inflammasome and NF- $\kappa \mathrm{B}$ signaling pathways. Thus, we speculated that EA-reduced TNF- $\alpha$ production might be associated with the regulation of the NLRP3 inflammasome/NF- $\kappa \mathrm{B}$ signaling.

To date, current PD therapy is focused on symptom control and fails to delay the progressive neurodegenerative process. Actually, various side effects of the available drugs present huge challenges for long-term application. Therefore, more potential therapeutic candidates are urgently essential for halting the progression of PD. Recent studies demonstrated that inhibition of neuroinflammation would 
attenuate DA neurodegeneration. Thus, anti-inflammatory agents might provide new avenues for PD treatment. However, the low success of translating promising antiinflammatory candidates from animal studies to clinical trials was indicated. Therefore, an urgent approach for a novel anti-inflammatory alternative design was prompted [28]. Since activation of the microglial NLRP3 inflammasome was verified to play a pivotal role in the progression of neurodegenerative disorders, such as AD, PD, and ALS, inhibition of NLRP3 inflammasome activation might become a promising therapeutic target for these neurodegenerative disorders. Here, the present study demonstrated that EA conferred DA neuroprotection against LPS-induced neurotoxicity and modulation of microglial NLRP3 inflammasome signaling activation was revealed to participate in this neuroprotection. These findings suggested that EA could open a new window on neurodegenerative disorder treatment. However, the current study on EA only stays in animal experiments, and the follow-up hopes to be used as a clinical drug application for future studies.

\section{Conclusion}

This study demonstrated that EA has a profound effect on protecting DA neurons against LPS-induced neurotoxicity via suppression of microglial NLRP3 inflammasome signaling activation. These findings suggest that EA might be a potential benefit for PD treatment.

\section{Abbreviations}

6-OHDA: 6-Hydroxydopamine

AD: Alzheimer's disease

DA: Dopamine

DMSO: Dimethyl sulfoxide

EA: $\quad$ Ellagic acid

ELISA: Enzyme-linked immunosorbent assay

FBS: $\quad$ Fetal bovine serum

Iba-1: $\quad$ Ionized calcium-binding adapter molecule-1

IL-1 $\beta$ : Interleukin- $1 \beta$

IL-18: Interleukin-18

LPS: $\quad$ Lipopolysaccharide

MCM: Microglia-conditioned medium

MEM: $\quad$ Minimum essential medium

NLRP3: Nod-like receptor protein 3

OX-42: Anti-CR3 complement receptor

PBS: $\quad$ Phosphate-buffered saline

PD: $\quad$ Parkinson's disease

PVDF: Polyvinylidene fluoride

siRNA: Small interfering RNA

SN: $\quad$ Substantia nigra

TH: $\quad$ Tyrosine hydroxylase

TNF- $\alpha$ : Tumor necrosis factor- $\alpha$.

\section{Data Availability}

Data in this manuscript were available from the corresponding author on reasonable request.

\section{Conflicts of Interest}

The authors declared no conflicts of interests.

\section{Authors' Contributions}

FZ conceived and designed the experiments. All the authors participated in the experiment performance and data analysis. FZ, YZZ, and XMH wrote, revised, and checked the article. All authors revised and approved the final manuscript. Xue-mei He and Yan-zhen Zhou contributed equally to this work.

\section{Acknowledgments}

This study was supported by the National Natural Science Foundation of China (No. 81760658), the Foundation for High-Level Innovative Talents of Guizhou Province (No. 20164027), the Innovation Research Group project of the Department of Education of Guizhou Province (No. 2016038), the Foundation for Excellent Young Talents of Zunyi Medical University (No. 201603), and the Master Start Foundation of Zunyi Medical University (No. F-898).

\section{References}

[1] Y. Zhang, V. L. Dawson, and T. M. Dawson, "Oxidative stress and genetics in the pathogenesis of Parkinson's disease," Neurobiology of Disease, vol. 7, no. 4, pp. 240-250, 2000.

[2] T. J. Collier, N. M. Kanaan, and J. H. Kordower, "Aging and Parkinson's disease: different sides of the same coin?," Movement Disorders, vol. 32, no. 7, pp. 983-990, 2017.

[3] A. J. Lees, J. Hardy, and T. Revesz, "Parkinson's disease," The Lancet, vol. 373, no. 9680, pp. 2055-2066, 2009.

[4] A. Raichur, S. Vali, and F. Gorin, "Dynamic modeling of alpha-synuclein aggregation for the sporadic and genetic forms of Parkinson's disease," Neuroscience, vol. 142, no. 3, pp. 859-870, 2006.

[5] L. Qian, P. M. Flood, and J. S. Hong, "Neuroinflammation is a key player in Parkinson's disease and a prime target for therapy," Journal of Neural Transmission (Vienna), vol. 117, no. 8, pp. 971-979, 2010.

[6] E. Hansson, "Long-term pain, neuroinflammation and glial activation," Scandinavian Journal of Pain, vol. 1, no. 2, pp. 67-72, 2010.

[7] F. Zhang, J. Liu, and J. S. Shi, "Anti-inflammatory activities of resveratrol in the brain: role of resveratrol in microglial activation," European Journal of Pharmacology, vol. 636, no. 1-3, pp. 1-7, 2010.

[8] W. Dauer and S. Przedborski, "Parkinson's disease: mechanisms and models," Neuron, vol. 39, no. 6, pp. 889-909, 2003.

[9] L. Franchi, R. Munoz-Planillo, and G. Nunez, "Sensing and reacting to microbes through the inflammasomes," Nature Immunology, vol. 13, no. 4, pp. 325-332, 2012.

[10] Y. He, H. Hara, and G. Nunez, "Mechanism and regulation of NLRP3 inflammasome activation," Trends in Biochemical Sciences, vol. 41, no. 12, pp. 1012-1021, 2016.

[11] M. T. Heneka, M. P. Kummer, A. Stutz et al., "NLRP3 is activated in Alzheimer's disease and contributes to pathology in APP/PS1 mice," Nature, vol. 493, no. 7434, pp. 674-678, 2013. 
[12] L. C. Freeman and J. P.-Y. Ting, "The pathogenic role of the inflammasome in neurodegenerative diseases," Journal of Neurochemistry, vol. 136, Supplement 1, pp. 29-38, 2016.

[13] F. Firdaus, M. F. Zafeer, E. Anis, M. Ahmad, and M. Afzal, "Ellagic acid attenuates arsenic induced neuro-inflammation and mitochondrial dysfunction associated apoptosis," Toxicology Reports, vol. 5, pp. 411-417, 2018.

[14] F. Moura, K. de Andrade, J. F. dos Santos, and M. F. Goulart, "Lipoic acid: its antioxidant and anti-inflammatory role and clinical applications," Current Topics in Medicinal Chemistry, vol. 15, no. 5, pp. 458-483, 2015.

[15] Q.-s. Liu, R. Deng, S. Li et al., "Ellagic acid protects against neuron damage in ischemic stroke through regulating the ratio of Bcl-2/Bax expression," Applied Physiology, Nutrition and Metabolism, vol. 42, no. 8, pp. 855-860, 2017.

[16] C. Latchoumycandane, V. Anantharam, H. Jin, A. Kanthasamy, and A. Kanthasamy, "Dopaminergic neurotoxicant 6-OHDA induces oxidative damage through proteolytic activation of PKC $\delta$ in cell culture and animal models of Parkinson's disease," Toxicology and Applied Pharmacology, vol. 256, no. 3, pp. 314-323, 2011.

[17] T. Wang, L. Wang, C. Li et al., "Hydroxysafflor yellow A improves motor dysfunction in the rotenone-induced mice model of Parkinson's disease," Neurochemical Research, vol. 42, no. 5, pp. 1325-1332, 2017.

[18] F. Zhang, J. S. Shi, H. Zhou, B. Wilson, J. S. Hong, and H. M. Gao, "Resveratrol protects dopamine neurons against lipopolysaccharide-induced neurotoxicity through its antiinflammatory actions," Molecular Pharmacology, vol. 78, no. 3, pp. 466-477, 2010.

[19] C. Chen, Y. Z. Wei, X. M. He et al., "Naringenin produces neuroprotection against LPS-induced dopamine neurotoxicity via the inhibition of microglial NLRP3 inflammasome activation," Frontiers in Immunology, vol. 10, p. 936, 2019.

[20] Y. Dong, M. L. Heien, M. M. Maxson, and A. G. Ewing, "Amperometric measurements of catecholamine release from single vesicles in MN9D cells," Journal of Neurochemistry, vol. 107, no. 6, pp. 1589-1595, 2008.

[21] J. van Meerloo, G. J. L. Kaspers, and J. Cloos, “Cell sensitivity assays: the MTT assay," Methods in Molecular Biology, vol. 731, pp. 237-245, 2011.

[22] S. Hirano, "Western blot analysis," Methods in Molecular Biology, vol. 926, pp. 87-97, 2012.

[23] J. G. Donaldson, "Immunofluorescence staining," Current Protocols in Cell Biology, vol. 69, no. 1, pp. 431-437, 2015.

[24] W. Shao, S. Z. Zhang, M. Tang et al., "Suppression of neuroinflammation by astrocytic dopamine D2 receptors via $\alpha \mathrm{B}$-crystallin," Nature, vol. 494, no. 7435, pp. 90-94, 2013.

[25] M. Jo, J. H. Kim, G. J. Song, M. Seo, E. M. Hwang, and K. Suk, "Astrocytic orosomucoid-2 modulates microglial activation and neuroinflammation," The Journal of Neuroscience, vol. 37, no. 11, pp. 2878-2894, 2017.

[26] G. Q. Wang, D. D. Li, C. Huang et al., "Icariin reduces dopaminergic neuronal loss and microglia-mediated inflammation in vivo and in vitro," Frontiers in Molecular Neuroscience, vol. 10, p. 441, 2018.

[27] C. S. Seo, S. J. Jeong, S. R. Yoo, N. R. Lee, and H. K. Shin, "Quantitative analysis and in vitro anti-inflammatory effects of gallic acid, ellagic acid, and quercetin from Radix Sanguisorbae," Pharmacognosy Magazine, vol. 12, no. 46, pp. 104-108, 2016.
[28] Y. Yan, W. Jiang, L. Liu et al., "Dopamine controls systemic inflammation through inhibition of NLRP3 inflammasome," Cell, vol. 160, no. 1-2, pp. 62-73, 2015.

[29] Z. L. Hu, T. Sun, M. Lu, J. H. Ding, R. H. du, and G. Hu, "Kir6.1/K-ATP channel on astrocytes protects against dopaminergic neurodegeneration in the MPTP mouse model of Parkinson's disease via promoting mitophagy," Brain, Behavior and Immunity, vol. 81, pp. 509-522, 2019.

[30] F. Di Virgilio, "The therapeutic potential of modifying inflammasomes and NOD-like receptors," Pharmacological Reviews, vol. 65 , no. 3, pp. 872-905, 2013.

[31] R. Gordon, E. A. Albornoz, D. C. Christie et al., "Inflammasome inhibition prevents $\alpha$-synuclein pathology and dopaminergic neurodegeneration in mice," Science Translational Medicine, vol. 10, no. 465, article eaah4066, 2018.

[32] E. Lee, I. Hwang, S. Park et al., "MPTP-driven NLRP3 inflammasome activation in microglia plays a central role in dopaminergic neurodegeneration," Cell Death and Differentiation, vol. 26, no. 2, pp. 213-228, 2019.

[33] B. Tang, G. X. Chen, M. Y. Liang, J. P. Yao, and Z. K. Wu, "Ellagic acid prevents monocrotaline-induced pulmonary artery hypertension via inhibiting NLRP3 inflammasome activation in rats," International Journal of Cardiology, vol. 180, pp. 134-141, 2015.

[34] W. Zhao, D. R. Beers, S. Bell et al., "TDP-43 activates microglia through NF- $\kappa \mathrm{B}$ and NLRP3 inflammasome," Experimental Neurology, vol. 273, pp. 24-35, 2015.

[35] L. Bai, J. Li, H. Li et al., "Renoprotective effects of artemisinin and hydroxychloroquine combination therapy on $\operatorname{IgA}$ nephropathy via suppressing NF- $\kappa \mathrm{B}$ signaling and NLRP3 inflammasome activation by exosomes in rats," Biochemical Pharmacology, vol. 169, article 113619, 2019. 